\title{
NUMERICAL MODELLING OF HEAT AND MAGNETOHYDRODYNAMIC FLOWS IN A FINITE CYLINDER
}

\author{
ANDRIS BUIKIS \\ Institute of Mathematics, Latvian Academy of Sciences and University of Latvia \\ 1, Akademijas sq., Riga, Latvia, LV-1524 \\ E-mail: buikis@latnet.Iv \\ HARIJS KALIS \\ Institute of Mathematics, Latvian Academy of Sciences and University of Latvia \\ 1, Akademijas sq., Riga, Latvia, LV-1524 \\ E-mail: kalis@latnet.Iv
}

\begin{abstract}
The distribution of electromagnetic fields, forces and temperatures induced by a three- phase axially-symmetric system of electric current in a conducting cylinder of finite length has been calculated. An original method was used to calculate the mean values of electromagnetic forces. The magnetohydrodynamic (MHD) flow of viscous incompressible liquid and distribution of temperature are obtained by the finite-difference method, using monotonic finite difference schemes.
\end{abstract}

2000 Mathematics Subject Classification: 65N06, 80A20.

Keywords: finite difference methods, heat and flows.

\section{Introduction}

In many technological applications it is important to mix and heat an electrically conducting liquid. In practice, such processes are implemented with the aid of various magnetic fields (rotating, traveling, and pulsing) acting upon the liquid.

One of the modern areas of application developed during last years is effective use of electric energy produced by alternating current in production of heat energy. This process is ecologically clean, there is no environment pollution. Though, on the other hand, the aspect of energy is very important: the transformation process is to be organized in such a way that electric energy should be effectively transformed into heat energy. It can be achieved by reducing the amount of elements in the energy transformation process. For example, if the medium, where heat energy is produced, is at the same time a transmitter in a heating system, then there will be no losses. One of such media is undistilled water. It is ecologically harmless and easily available. It is very important that water (with a small amount of nonaggressive salts added, if needed) is a weakly electrically conducting medium (electrolyte). 
In the last ten years, devices based on this principle have been developed. Compared to the classical devices with heating elements, they are more compact and do not calcify.

This work presents a mathematical model of one of such devices. It is a finite cylinder with equally spaced metal coils on its inner surface. On connecting these coils to a one-phase or three-phase alternating current, they start to rebase energy. If this cylinder is placed, for example, in a house heating system together with a small electric motor which rotates water at the inlet of the cylinder and pumps water through it, we obtain an effective, compact, and ecologically clean house heating device.

\section{Main assumption of the mathematical model}

Magnetic fields in a conducting medium are induced owing to the following two factors:

1) on interaction of an electromagnetic field with the electrically conducting medium;

2) on interaction of a moving medium with an external magnetic field.

In the general case, both factors could be sufficiently important. However, if the estimation $e=\mu \sigma \tilde{\omega} a<<1$ is valid, then the second factor can be ignored. Here, $\mu=4 \pi 10^{-7} \frac{\mathrm{m} . \mathrm{kg}}{\mathrm{s}^{2} \cdot \mathrm{A}^{2}}$ is the magnetic permeability in vacuum, $\sigma$ is the electric conductivity of the liquid, $\tilde{\omega}=2 \pi f$ is the angular frequency of the alternating current fed to the installation, $f$ is the frequency, and $a$ is the cylinder radius. For an electrolyte with $\sigma=100 \frac{1}{\Omega . m}$ at $a=0.05 \mathrm{~m}$ and $f=50 \mathrm{~Hz}$ we obtain $e=10^{-4}<<1$. This means that the action of the moving liquid on the external magnetic field is insignificant, that is, the process could be considered in the so-called inductionless approximation. This allows the current density vector $j$ to be defined from Ohm's law in the simplified form $j=\sigma E$, where $E$ is the intensity vector of the alternating electric field.

Let the cylindrical domain $\tilde{\Omega}=\{(r, z): 0<r<a, 0<z<Z\}$ contain conducting material, where $a, Z$ are the radius and length of the cylinder. Consider the situation when an alternating current with the density $j_{i}=j_{0} \cos (\tilde{\omega} t+(i-1) \theta), i=\overline{1, N}$, is fed to $N$ discrete circular conductors, which are placed on the internal wall of the cylinder. Here $j_{0}$ is the amplitude, $\theta$ is the phase (usually $\theta=60^{\circ}$ ), and $t$ is the time. In the conducting liquidelectrolyte the current creates the radial $B_{r}$ and axial $B_{z}$ components of the magnetic field as well as the azimuthal component of the induced electric field $E_{\phi}$, which, in turn, creates the axial $F_{z}$ and radial $F_{r}$ components of the electromagnetic force (Lorentz' force). To calculate the electromagnetic fields, the averaging method over the time interval $2 \pi / \tilde{\omega}=1 / f$ was used. The values $F_{r}, F_{z}$ of these latter components averaged within the time interval $f^{-1}$ give rise to the liquid (electrolyte) motion, which can be described by the stationary Navier-Stokes equation. At the inlet of the cylinder we have a uniform velocity $U_{0} \approx 0.1 \frac{\mathrm{m}}{\mathrm{s}}$, but the swirl velocity is taken to be that induced by the rigid body rotation with the angular velocity $\Omega_{0} \approx 4 \frac{1}{s}$. In $[3,5]$, numerical solutions have been obtained for a swirling flow of a viscous incompressible fluid under various boundary constraints. In [5], a rotating flow is obtained mechanically, but in [3] it was induced by a magnetic field and direct current. The direct current is fed to the electrodes located in the entry section of the cylinder. Our work deals with electrodes positioned on the inner surface of the cylinder having a contact with a moving medium. Magnetic field is produced by passing an alternating current to the electrodes. The main aim of the work is to analyze the way in which different connection schemes of the electrodes and vortices influence the maximum value of temperature.

Most of the works devoted to investigation into the influence of alternating current on the distribution of electromagnetic forces deal with an infinitely long inductor. A quasi- 
stationary magnetic field is calculated based on the form of Bessel's functions and complex number with the factor $\exp \left(j^{*} \tilde{\omega} t\right)$, where $j^{*}=\sqrt{-1}[4]$. In the present work, which employs elliptical integrals, the calculations are performed using only the real form of numbers and assuming the time dependence as $\cos (\tilde{\omega} t)$. This makes it possible to consider alternating current connections of various type, with phase shifts $\theta$ and various arrangements of conductors.

The liquid has the following parameters: kinematic viscosity $\nu \approx 10^{-5} \frac{m^{2}}{s}$, density $\rho \approx$ $1000 \frac{\mathrm{kg}}{\mathrm{m}^{3}}(\eta=\rho \nu$ is the dynamic viscosity $)$, specific heat capacity $c \approx 4000 \frac{\mathrm{J}}{\mathrm{kg} \cdot \mathrm{K}}$, heat conductivity $\lambda \approx 0.6 \frac{W}{m \cdot K}$, and the heat exchange coefficient $\alpha \approx 12 \frac{w}{m^{2} \cdot K}$.

\section{Calculation of the electromagnetic field and force}

The azimuthal components $A_{\phi}, E_{\phi}$ of the vector potential $A$ and of the electric field $E$ are determined from Maxwell's equations in the following form:

$$
E_{\phi}=-\partial A_{\phi} / \partial t ; \quad \Delta A_{\phi}=-\mu j_{\phi}^{e},
$$

where $B=\operatorname{rot} A=\mu H$ and $H, B, j^{e}$ are the vectors of the magnetic field intensity, induction, and the vector of the external current density, $\Delta$ is the Laplace operator.

Applying the Biot-Savar law, we arrive at the following form of the azimuthal component of the vector potential created by the alternating current in every conductor $L_{i}$ :

$$
A_{\phi, i}(t, r, z)=\frac{\mu j_{i}}{4 \pi} \int_{L_{i}} \frac{d l}{\sqrt{\left(\left(z-z_{i}\right)^{2}+(r-a)^{2}\right)}},
$$

where $d l$ is an element of the circular line. Having evaluated the line integrals in a closed form we have

$$
A_{\phi, i}(t, r, z)=\frac{\mu j_{0}}{2 \pi} A_{i}(r, z) \cos (\tilde{\omega} t+(i-1) \theta)
$$

where

$$
\begin{aligned}
A_{i}(r, z) & =\sqrt{\frac{a}{r}}\left[\left(\frac{2}{k_{i}}-k_{i}\right) K\left(k_{i}\right)-\frac{2}{k_{i}} E\left(k_{i}\right)\right], \quad k_{i}=\frac{2 \sqrt{a r}}{c_{i}}, \quad c_{i}=\sqrt{(a+r)^{2}+\left(z-z_{i}\right)^{2}}, \\
K(k) & =\int_{0}^{\pi / 2} \frac{d \xi}{\sqrt{1-k^{2} \sin ^{2} \xi}} \text { is the total elliptical integral of first kind, } \\
E(k) & =\int_{0}^{\pi / 2} \sqrt{1-k^{2} \sin ^{2} \xi} d \xi \text { is the total elliptical integral of second kind. This integrals }
\end{aligned}
$$
can be calculated accurate to the 4-th decimal place [1].

Assuming the vectorial components of the magnetic field induction as $B_{r}=-\partial A_{\phi} / \partial z$, $B_{z}=\frac{1}{r} \frac{\partial}{\partial r}\left(r A_{\phi}\right)$, we arrive at the following form of these components created by the alternating current in the conductor $L_{i}$ :

$$
\begin{aligned}
& B_{r, i}(t, r, z)=\frac{\mu j_{0}}{2 \pi} B_{i}^{r}(r, z) \cos (\tilde{\omega} t+(i-1) \theta), \\
& B_{z, i}(t, r, z)=\frac{\mu j_{0}}{2 \pi} B_{i}^{z}(r, z) \cos (\tilde{\omega} t+(i-1) \theta),
\end{aligned}
$$


where

$$
\begin{aligned}
& B_{i}^{r}(r, z)=\sqrt{\frac{a}{r}} \frac{z-z_{i}}{c_{i}}\left[E\left(k_{i}\right) \frac{a^{2}+r^{2}+\left(z-z_{i}\right)^{2}}{(a-r)^{2}+\left(z-z_{i}\right)^{2}}-K\left(k_{i}\right)\right], \\
& B_{i}^{z}(r, z)=\frac{1}{c_{i}}\left[K\left(k_{i}\right)+\frac{a^{2}-r^{2}-\left(z-z_{i}\right)^{2}}{(a-r)^{2}+\left(z-z_{i}\right)^{2}} E\left(k_{i}\right)\right] .
\end{aligned}
$$

From Ohm's law for the electrically conducting media follows the azimuthal components $j_{\phi}$ of the vector of the induced current density as $j_{\phi}=\sigma E_{\phi}=-\sigma \partial A_{\phi} / \partial t$, and hence from every conductor $L_{i}$ electric current with the density

$$
j_{\phi, i}(t, r, z)=\frac{\tilde{\omega} \mu \sigma j_{0}}{2 \pi} A_{i}(r, z) \sin (\tilde{\omega} t+(i-1) \theta)
$$

is induced.

The summary magnetic field $\left(B_{r}, B_{z}\right)$, the vector potential $A_{\phi}$, and the induced current density $j_{\phi}$ we obtain from (3.1-3.3) in the form

$$
B_{r}=\sum_{i=1}^{N} B_{r, i}, \quad B_{z}=\sum_{i=1}^{N} B_{z, i}, \quad A_{\phi}=\sum_{i=1}^{N} A_{\phi, i}, \quad j_{\phi}=\sum_{i=1}^{N} j_{\phi, i} .
$$

If $t=0$, then from (3.4) one can evaluate the instantaneous values of the above quantities.

Having calculated the vector of the electromagnetic force $F=j \times B$, we can write the instantaneous value of the radial and axial components as $F_{r}=B_{z} j_{\phi}, F_{z}=-B_{r} j_{\phi}$.

Therefore,

$$
\begin{aligned}
& F_{r}(t, r, z)=\left(\frac{\mu j_{0}}{2 \pi}\right)^{2} \sigma \tilde{\omega} \sum_{i, j=1}^{N} \alpha_{i, j}^{r}(r, z) c s(t), \\
& F_{z}(t, r, z)=\left(\frac{\mu j_{0}}{2 \pi}\right)^{2} \sigma \tilde{\omega} \sum_{i, j=1}^{N} \alpha_{i, j}^{z}(r, z) c s(t),
\end{aligned}
$$

where

$$
\begin{aligned}
c s(t) & =\cos (\tilde{\omega} t+(i-1) \theta) \sin (\tilde{\omega} t+(j-1) \theta) \\
\alpha_{i, j}^{r}(r, z) & =B_{i}^{z} A_{j}=\frac{A_{i} A_{j}}{r}+\frac{A_{j} \partial A_{i}}{\partial r}=\alpha_{i, j}^{r s}+\alpha_{i, j}^{r a}, \\
\alpha_{i, j}^{z}(r, z) & =-B_{i}^{r} A_{j}=\frac{A_{j} \partial A_{i}}{\partial z}=\alpha_{i, j}^{z s}+\alpha_{i, j}^{z a}, \\
\alpha_{i, j}^{z s} & =0.5 \frac{\partial\left(A_{i} A_{j}\right)}{\partial z}, \quad \alpha_{i, j}^{z a}=0.5\left(\frac{A_{j} \partial A_{i}}{\partial z}-\frac{A_{i} \partial A_{j}}{\partial z}\right), \\
\alpha_{i, j}^{r s} & =0.5 \frac{\partial\left(A_{i} A_{j}\right)}{\partial r}+\frac{A_{i} A_{j}}{r}, \quad \alpha_{i, j}^{r a}=0.5\left(\frac{A_{j} \partial A_{i}}{\partial r}-\frac{A_{i} \partial A_{j}}{\partial r}\right) .
\end{aligned}
$$

Note that the symmetrical and antisymmetrical parts of the coefficients $\alpha$ are satisfied by the relationships $\alpha_{i, j}^{z s}=\alpha_{j, i}^{z s}, \alpha_{i, j}^{r s}=\alpha_{j, i}^{r s}, \alpha_{i, j}^{z a}=-\alpha_{j, i}^{z a}, \quad \alpha_{i, j}^{r a}=-\alpha_{j, i}^{r a}$.

Since $c s(t)=0.5(\sin (2 \tilde{\omega} t+(i+j-2) \theta)+\sin (\theta(j-i)))$, the average quantity in the time interval $2 \pi / \tilde{\omega}$ of this expression is $0.5 \sin ((j-i) \theta)$. Therefore, the radial and axial components of the averaged force vector are written as

$$
<F_{r}>=0.5\left(\frac{\mu j_{0}}{2 \pi}\right)^{2} \sigma \tilde{\omega} S_{N}^{r}, \quad<F_{z}>=0.5\left(\frac{\mu j_{0}}{2 \pi}\right)^{2} \sigma \tilde{\omega} S_{N}^{z},
$$


where

$$
\begin{aligned}
& S_{N}^{r}=\sum_{i, j=1}^{N} \sin ((j-i) \theta) \alpha_{i, j}^{r}=2 \sum_{k=1}^{N-1} \sin (k \theta) \sum_{i=1}^{N-k} \alpha_{i, k+i}^{r a}, \\
& S_{N}^{z}=\sum_{i, j=1}^{N} \sin ((j-i) \theta) \alpha_{i, j}^{z}=2 \sum_{k=1}^{N-1} \sin (k \theta) \sum_{i=1}^{N-k} \alpha_{i, k+i}^{z a} .
\end{aligned}
$$

Having calculated the azimuthal component of the curl of the force vector $f_{\phi}=\partial F_{r} / \partial z-$ $\partial F_{z} / \partial r=\partial\left(B_{z} j_{\phi}\right) / \partial z+\partial\left(B_{r} j_{\phi}\right) / \partial r$, we analogously obtain its average value as

$$
<f_{\phi}>=0.5\left(\frac{\mu j_{0}}{2 \pi}\right)^{2} \sigma \tilde{\omega} S_{N}^{\phi}
$$

where

$$
\begin{aligned}
S_{N}^{\phi} & =\sum_{i, j=1}^{N} \sin ((j-i) \theta) \alpha_{i, j}^{\phi}=2 \sum_{k=1}^{N-1} \sin (k \theta) \sum_{i=1}^{N-k} \alpha_{i, k+i}^{\phi a}, \\
\alpha_{i, j}^{\phi}(r, z) & =\frac{\partial\left(B_{i}^{z} A_{j}\right)}{\partial z}+\frac{\partial\left(B_{i}^{r} A_{j}\right)}{\partial r}=\alpha_{i, j}^{\phi s}+\alpha_{i, j}^{\phi a}, \\
\alpha_{i, j}^{\phi s} & =\alpha_{j, i}^{\phi s}=\frac{1}{r} \frac{\partial}{\partial z}\left(A_{i} A_{j}\right), \quad \alpha_{i, j}^{\phi a}=-\alpha_{j, i}^{\phi a}=\frac{\partial A_{i}}{\partial r} \frac{\partial A_{j}}{\partial z}-\frac{\partial A_{i}}{\partial z} \frac{\partial A_{j}}{\partial r} .
\end{aligned}
$$

Calculating the derivatives gives

$$
\begin{aligned}
& \frac{\partial A_{i}}{\partial r}=\frac{1}{c_{i}}\left[\left(1-\frac{a^{2}+r^{2}+\left(z-z_{i}\right)^{2}}{r^{2}}\right) K\left(k_{i}\right)+\left(\frac{a^{2}-r^{2}-\left(z-z_{i}\right)^{2}}{\left.(a-r)^{2}+z-z_{i}\right)^{2}}+\frac{c_{i}^{2}}{r^{2}}\right) E\left(k_{i}\right)\right], \\
& \frac{\partial A_{i}}{\partial z}=-B_{i}^{r} .
\end{aligned}
$$

The function (3.7) is employed in calculating the motion of liquids according to the NavierStocks equation. It is readily seen that $\partial \alpha_{i, j}^{r a} / \partial z-\partial \alpha_{i, j}^{z a} / \partial r=\alpha_{i, j}^{\phi a}$.

\section{The Navier-Stokes equations.}

The complete system of equations for a viscous incompressible flow consists of the continuity equation, Navier-Stokes equation and energy (temperature) equation. The axially-symmetric stationary Navier-Stokes equations for a viscous incompressible flow in the cylindrical coodinates $(r, \phi, z)$ have the following form:

$$
\left\{\begin{aligned}
M\left(v_{z}\right) & =-\rho^{-1} \frac{\partial p}{\partial z}+\nu \Delta v_{z}+\rho^{-1}<F_{z}> \\
M\left(v_{r}\right) & =-\rho^{-1} \frac{\partial p}{\partial r}+\nu\left(\Delta v_{r}-r^{-2} v_{r}\right)+r^{-1} v_{\phi}^{2}+\rho^{-1}<F_{r}> \\
M\left(v_{\phi}\right)+r^{-1} v_{r} v_{\phi} & =\nu\left(\Delta v_{\phi}-r^{-2} v_{\phi}\right) \\
r^{-1} \frac{\partial\left(r v_{r}\right)}{\partial r}+\frac{\partial v_{z}}{\partial z} & =0
\end{aligned}\right.
$$


where $p$ is the pressure, $\Delta(g)=r^{-1} \partial(r \partial g / \partial r) / \partial r+\partial^{2} g / \partial z^{2}$ is the expression of Laplace operator. Here, the radial, axial, and azimuthal components of the velocity are denoted by $v_{r}, v_{z}$, and $v_{\phi}$ respectively, while $M(g)=v_{z} \frac{\partial g}{\partial z}+v_{r} \frac{\partial g}{\partial r}$ are the convective terms of the equations. Taking the derivatives of the first equation of the system (4.1) with respect to $r$ and subtracting it from the second equation, which is differentiated with respect to $z$, we can exclude the pressure. Then, the first two equations yield

$$
M\left(\omega_{\phi}\right)-r^{-1} v_{r} \omega_{\phi}=\nu\left(\Delta \omega_{\phi}-r^{-2} \omega_{\phi}\right)+r^{-1} \frac{\partial v_{\phi}^{2}}{\partial z}+\rho^{-1}<f_{\phi}>,
$$

where $\omega_{\phi}=\frac{\partial v_{r}}{\partial z}-\frac{\partial v_{z}}{\partial r}$ is the azimuthal component of the velocity curl vector, $<f_{\phi}>=$ $\frac{\partial\left\langle F_{r}\right\rangle}{\partial z}-\frac{\partial\left\langle F_{z}\right\rangle}{\partial r}$ is the averaged azimuthal component of the force curl vector.

To ensure fulfillment of the forth (continuous) equation of system (4.1), we will define the hydrodynamic-stream function $\psi$ with the aid of the relationships

$$
v_{r}=-r^{-1} \frac{\partial \psi}{\partial z}, v_{z}=r^{-1} \frac{\partial \psi}{\partial r}
$$

Then,

$$
\omega_{\phi}=-r^{-1} \Delta^{*}(\psi),
$$

where $\Delta^{*}(g)=r \partial\left(r^{-1} \partial g / \partial r\right) / \partial r+\partial^{2} g / \partial z^{2}$ is the conjugate expression for the Laplace operator. Assuming that $\Delta \omega_{\phi}-r^{-2} \omega_{\phi}=r^{-1} \Delta^{*}\left(r \omega_{\phi}\right), M\left(\omega_{\phi}\right)-r^{-1} \omega_{\phi} v_{r}=r M\left(r^{-1} \omega_{\phi}\right)$, equation (4.2) can be written in the form

$$
r M\left(\omega_{\phi} / r\right)=\nu r^{-1} \Delta^{*}\left(r \omega_{\phi}\right)+r^{-1} \frac{\partial v_{\phi}^{2}}{\partial z}+\rho^{-1}<f_{\phi}>
$$

which, together with (4.4), forms the closed system of equations for determining the functions $\psi, \omega_{\phi}$. To form a monotonous finite-difference scheme [2], it is necessary that equations (4.5), could contain only partial derivatives of the functions $\psi, \omega_{\phi}$, and $v_{\phi}$. For this purpose, we will use the transformations

$$
\omega=r^{-1} \omega_{\phi}, \quad W=r v_{\phi} .
$$

Then, the equation for the vorticity function $\omega$, stream function $\psi$, and the circulation $W$ will be written in the following form:

$$
\left\{\begin{aligned}
\frac{J(\psi, \omega)}{r} & =\nu\left(\partial^{2} \omega / \partial z^{2}+r^{-3} \partial\left(r^{3} \partial \omega / \partial r\right) / \partial r\right)+2 W r^{-4} \partial W / \partial z+\frac{<f_{\phi}>}{\rho r} \\
r^{-1} J(\psi, W) & =\nu \Delta^{*}(W) \\
\Delta^{*}(\psi) & =-r^{2} \omega
\end{aligned}\right.
$$

where

$J(\psi, b)=\frac{\partial \psi}{\partial r} \frac{\partial b}{\partial z}-\frac{\partial b}{\partial r} \frac{\partial \psi}{\partial z}$ is the Jacobian of the functions $\psi$ and $b$ by $b=\omega$ or $b=W$. The boundary conditions have the following form:

1) at the inlet $\left(z=0,0 \leqslant r<r_{1}\right)$ the axial streams are assumed to have a uniform velocity $\left(v_{z}=U_{0}, W=\omega=0, \psi=0.5 U_{0} r^{2}\right.$; in the remaining part of the inlet section $\left(z=0, r_{1} \leqslant r \leqslant a\right)$ the swirl velocity profile is taken as that induced by the rigid body rotation with the angular velocity $\Omega_{0}, W=W_{0} r^{2}\left(W_{0}=\Omega_{0} a^{2}\right), \omega=0, \psi=$ $0.5 U_{0}\left(r_{1}^{2}+\beta\left(r^{2}-r_{1}^{2}\right)\right)$, where $\beta \approx 0.1$ is the velocity ratio of the to the axial free stream velocity to axial jet velocity $U_{0}$, 
2) the symmetry conditions along the axis $(r=0), \psi=W=\partial \psi / \partial r=\partial \omega / \partial r=0$,

3) the outflow boundary conditions at the outlet $(z=Z), \partial \psi / \partial z=\partial \omega / \partial z=\partial W / \partial z=0$,

4) the walls boundary conditions $(r=a), W=0, \psi_{w}=0.5 U_{0}\left(r_{1}^{2}+\beta\left(a^{2}-r_{1}^{2}\right)\right), \omega=\omega_{w}$, where $\omega_{w}$ is the wall-vorticity, obtained in the finite-difference method from no-slip conditions $\left(v_{r}=v_{z}=0\right)$.

The equations (4.7) and the boundary conditions were nondimensionalized by referring all the lengths to $r_{0}=a$ (the inlet radius of the tube), the axial velocity $\left(v_{z}\right)$ to $U_{0}$ ( the uniform inlet axial velocity), swirl velocity $(W)$ to $W_{0}=r_{0} V_{0}$, the azimuthal velocity $\left(v_{\phi}\right)$ to $V_{0}=r_{0} \Omega_{0}$, the vorticity $(\omega)$ to $\omega_{0}=U_{0} /\left(r_{0}^{2}\right)$, and the stream function $(\psi)$ to $\psi_{0}=U_{0} r_{0}^{2}$.

The nondimensional equations are

$$
\left\{\begin{aligned}
\frac{R e}{r} J(\psi, \omega) & =\frac{\partial^{2} \omega}{\partial z^{2}}+r^{-3} \frac{\partial}{\partial r}\left(r^{3} \frac{\partial \omega}{\partial r}\right)+2 R e \Gamma^{2} W r^{-4} \frac{\partial W}{\partial z}+\frac{R e . T e . f^{\phi}}{r} \\
\frac{R e}{r} J(\psi, W) & =\Delta^{*}(W) \\
\Delta^{*}(\psi) & =-r^{2} \omega
\end{aligned}\right.
$$

where $\Gamma=V_{0} / U_{0} \approx 2$ is the swirl number, $f^{\phi}=0.5 S_{N}^{\phi}$ is the nondimensional value of $\left\langle f_{\phi}>\right.$, $R e=U_{0} r_{0} / \nu \approx 500$ is the Reynolds number, and $T e=\sigma \tilde{\omega}\left(\frac{\mu j_{0}}{2 \pi U_{0}}\right)^{2} / \rho \approx 10^{-10} j_{0}^{2}$ is the Taylor number. If the axial stream vanishes, then we have $U_{0}=\nu / r_{0}$ and $R e=1$.

We have the following boundary condition in dimensionless form:

1) for $\left(z=0,0 \leqslant r<r_{1}\right), \quad W=\omega=0, \psi=0.5 r^{2}$; for $\left(z=0, r_{1} \leqslant r \leqslant 1\right), \quad W=r^{2}, \omega=0, \psi=0.5\left(r_{1}^{2}+\beta\left(r^{2}-r_{1}^{2}\right)\right)$,

2) for $(r=0), \quad \psi=W=\partial \psi / \partial r=\partial \omega / \partial r=0$,

3) for $(z=l=Z / a), \quad \partial \psi / \partial z=\partial \omega / \partial z=\partial W / \partial z=0$,

4) for $(r=1), \quad W=0, \psi_{w}=0.5\left(r_{1}^{2}+\beta\left(1-r_{1}^{2}\right)\right), \omega=\omega_{w}$, where $\omega_{w}$ is the dimensionless wall vorticity, obtained from the no-slip conditions, and $r, r_{1}$ are the dimensionless coordinates.

\section{The heat transfer equation}

In an incompressible flow with constant properties the hydrodynamics is independent of the thermodynamics. Once the kinematic flow field is described by $\psi, \omega$, and by $W$ solutions, we can obtain solution for any number of temperature distributions $T$ with different thermal boundary conditions. The steady energy equation reduces to the heat transport equation for an incompressible flow with source terms and with constant properties. The axially-symmetric stationary distribution of the temperature field in a conducting cylinder is described by the following equation of heat conduction in cylindrical coordinates:

$$
v_{z} \partial T / \partial z+v_{r} \partial T / \partial r=\chi \Delta T+c^{-1} \nu \Phi+(\sigma \rho c)^{-1}<j_{\phi}^{2}>
$$


where $\left\langle j_{\phi}^{2}>\right.$ is the averaged value of $j_{\phi}^{2}$ (the source function), $T=T(r, z)$ is the steady-state distribution of the absolute temperature in a conducting medium, $\chi=\lambda /(c \rho) \approx 0.1510^{-6} \frac{\mathrm{m}^{2}}{\mathrm{~s}}$ is the thermal diffusivity,

$$
\begin{aligned}
\Phi= & 2\left(\left(\frac{\partial v_{r}}{\partial r}\right)^{2}+\left(\frac{\partial v_{z}}{\partial z}\right)^{2}+\frac{1}{r^{2}}\left(\frac{\partial v_{\phi}}{\partial \phi}+v_{r}\right)^{2}\right)+\left(\frac{\partial v_{r}}{\partial z}+\frac{\partial v_{z}}{\partial r}\right)^{2} \\
& +\left(\frac{\partial v_{\phi}}{\partial z}+\frac{1}{r} \frac{\partial v_{z}}{\partial \phi}\right)^{2}+\left(\frac{\partial v_{\phi}}{\partial r}+\frac{1}{r}\left(\frac{\partial v_{r}}{\partial \phi}-v_{\phi}\right)\right)^{2}
\end{aligned}
$$

is the dissipation function in cylindrical coordinates.

Using (3.3)-(3.4) and the expression

$$
\sin (\tilde{\omega} t+(j-1) \theta) \sin (\tilde{\omega} t+(i-1) \theta)=0.5(-\cos (2 \tilde{\omega} t+(i+j-2) \theta)+\cos (\theta(j-i))),
$$

we have

$$
<j_{\phi}^{2}>=0.5\left(\frac{\mu j_{0} \sigma \tilde{\omega}}{2 \pi}\right)^{2} S_{N}^{Q}
$$

where

$$
\begin{aligned}
S_{N}^{Q} & =\sum_{i, j=1}^{N} \cos ((j-i) \theta) \alpha_{i, j}^{Q}=2 \sum_{k=1}^{N-1} \cos (k \theta) \sum_{i=1}^{N-k} \alpha_{i, k+i}^{Q}+\sum_{i=1}^{N} \alpha_{i, i}^{Q}, \\
\alpha_{i, j}^{Q} & =\alpha_{j, i}^{Q}=A_{i} A_{j} .
\end{aligned}
$$

To formulate the boundary-value problem for equation (5.1), the boundary conditions are written in the form

$$
\left.\lambda \frac{\partial T}{\partial r}\right|_{r=a}=\alpha\left(T_{a}-\left.T\right|_{r=a}\right),\left.\quad T\right|_{z=0}=T_{a},\left.\quad \frac{\partial T}{\partial z}\right|_{z=Z}=\left.\frac{\partial T}{\partial r}\right|_{r=0}=0,
$$

where $T_{a}$ is the given constant external temperature.

Introducing the dimensionless values $\tilde{T}=\left(T-T_{a}\right) / T_{a}, \tilde{r}=r / a, \tilde{z}=Z / a$, we arrive at the following form of the boundary-value problem for the heat transport equation in cylindrical coordinates (the symbol "tilde" is omitted):

$$
\left\{\begin{array}{c}
\frac{P e}{r} J(\psi, T)=\Delta T+\operatorname{Ec} \operatorname{Pr} \Phi+K_{T} q \\
\left.\frac{\partial T}{\partial r}\right|_{r=1}=-\left.B i T\right|_{r=1},\left.\quad T\right|_{z=0}=0,\left.\quad \frac{\partial T}{\partial z}\right|_{z=l}=\left.\frac{\partial T}{\partial r}\right|_{r=0}=0
\end{array}\right.
$$

where $K_{T}=\frac{\left(\mu j_{0} \tilde{\omega} a\right)^{2} \sigma}{(2 \pi)^{2} \lambda T_{a}} \approx 0.510^{-11} j_{0}^{2}$ is the heat sources parameter, $B i=\frac{\alpha a}{\lambda} \approx 1$ is the Biot number,

$$
\begin{aligned}
q= & 0.5 S_{N}^{Q} \\
\Phi= & 2\left(\left(\frac{\partial}{\partial r}\left(\frac{1}{r} \frac{\partial \psi}{\partial z}\right)\right)^{2}+\left(\frac{\partial}{\partial z}\left(\frac{1}{r} \frac{\partial \psi}{\partial r}\right)\right)^{2}+\left(\frac{1}{r^{2}} \frac{\partial \psi}{\partial z}\right)^{2}\right) \\
& +\left(\frac{\partial}{\partial r}\left(\frac{1}{r} \frac{\partial \psi}{\partial r}\right)-\frac{1}{r} \frac{\partial^{2} \psi}{\partial z^{2}}\right)^{2}+\Gamma^{2}\left(\left(\frac{1}{r} \frac{\partial W}{\partial z}\right)^{2}+\left(r \frac{\partial\left(\frac{W}{r^{2}}\right)}{\partial r}\right)^{2}\right)
\end{aligned}
$$

is the dimensionless axially-symmetric dissipation function, $\operatorname{Pr}=c \rho \nu / \lambda=\nu / \chi \approx 67, \operatorname{Pe}=$ $U_{0} a / \chi \approx 10^{4}, E c=U_{0}^{2} /\left(c T_{a}\right) \approx 10^{-8}$ are the Prandtl, Peclet, and Eckert numbers $(P e=$ $\operatorname{Pr} . R e)$. 


\section{The wall vorticity in a uniform grid}

We consider a uniform grid $\left(n_{r} \times n_{z}\right): \omega_{h}=\left\{\left(r_{i}, z_{j}\right), r_{i}=0.5 h_{1}+(i-1) h_{1}, z_{j}=(j-1) h_{2}, i=\right.$ $\left.\overline{1, n_{r}}, j=\overline{1, n_{z}}, n_{r} h_{1}=1, n_{z} h_{2}=l\right\}$. The subscripts $(i, j)$ refer to the $r, z$ indices, the mesh spacing in the $i, j$ directions are $h_{1}$ and $h_{2}$. We use the same notation $g$ for the continuum function $g(r, z)$ and the discrete function $g\left(r_{i}, z_{j}\right)=g_{i, j}$.

The vorticity $\omega_{w}$ is obtained from the no-slip conditions [6]. We expand to $\psi_{n_{r}, j}$ by a Taylor series out from the wall values $\psi_{m, j}\left(m=n_{r}+0.5, m h_{1}=1\right)$ :

$$
\psi_{n_{r}, j}=\psi_{m, j}-\frac{h_{1}}{2}\left(\frac{\partial \psi}{\partial r}\right)_{m, j}+\frac{h_{1}^{2}}{8}\left(\frac{\partial^{2} \psi}{\partial r^{2}}\right)_{m, j}-\frac{h_{1}^{3}}{48}\left(\frac{\partial^{3} \psi}{\partial r^{3}}\right)_{m, j}+O\left(h_{1}^{4}\right) .
$$

But $\psi_{m, j}=\psi_{w},\left(\frac{\partial \psi}{\partial r}\right)_{m, j}=\left(v_{z}\right)_{m, j}=0$ according to the no-slip condition, and $\left(\frac{\partial^{2} \psi}{\partial r^{2}}\right)_{m, j}=$ $\left(v_{z}+r \frac{\partial v_{z}}{\partial r}\right)_{m, j}$. Now, $\quad \omega=\frac{1}{r}\left(\frac{\partial v_{r}}{\partial z}-\frac{\partial v_{z}}{\partial r}\right)$. Along the wall $\left(\frac{\partial v_{r}}{\partial z}\right)_{m, j}=0$, because $v_{r}=0$ along $z$. Thus, $\left(\frac{\partial v_{z}}{\partial r}\right)_{m, j}=-\omega_{w}=\left(\frac{\partial^{2} \psi}{\partial r^{2}}\right)_{m, j}, \omega_{w}=\omega_{m, j}$. Substituting this into equation (6.1) and solving for $\omega_{m, j}$ give

$$
\omega_{w}=-\frac{8}{h_{1}^{2}}\left(\psi_{n_{r}, j}-\psi_{w}\right)+O\left(h_{1}\right) .
$$

This first-order form was given by Thom (1928). Woods (1954) suggested the next secondorder form, obtained from equation (6.1) by retaining the $O\left(h_{1}^{3}\right)$ terms. By differentiating the definition of vorticity, we obtain $\left(\frac{\partial \omega}{\partial r}\right)_{m, j}=3\left(\frac{\partial^{2} \psi}{\partial r^{2}}\right)_{m, j}-\left(\frac{\partial^{3} \psi}{\partial r^{3}}\right)_{m, j}=-3 \omega_{w}-\left(\frac{\partial^{3} \psi}{\partial r^{3}}\right)_{m, j}$ (the term $\frac{\partial^{3} \psi}{\partial r \partial z^{2}}$ is zero according to the non-slip condition). The term $\left(\frac{\partial \omega}{\partial r}\right)_{m, j}$ is evaluated by a first-order backward difference as $\left(\frac{\partial \omega}{\partial r}\right)_{m, j}=\left(\omega_{w}-\omega_{n_{r}, j}\right) /\left(0.5 h_{1}\right)+O\left(h_{1}\right)$. Substituting the equation for $\left(\frac{\partial^{3} \psi}{\partial r^{3}}\right)_{m, j}$ into equation (6.1)and solving for $\omega_{m, j}$ give the Woods method for wall vorticity

$$
\omega_{w}=\left(-\frac{12}{h_{1}^{2}}\left(\psi_{n_{r}, j}-\psi_{w}\right)-\frac{1}{2} \omega_{n_{r}, j}\right) /\left(1-0.75 h_{1}\right)+O\left(h_{1}^{2}\right) .
$$

From (6.2), (6.3) it follows that

$$
\omega_{n_{r}+1, j}=2 \omega_{w}-\omega_{n_{r}, j}
$$

\section{Finite-difference approximations for 1-D model equations}

The presence of large parameters of first order derivatives $(\Gamma, R e, P e)$ in the system of differential equations (4.8), (5.4) couses additional numerical difficulties for application of the general finite-difference methods (little speed of convergence, low precision). Thus, special monotonic approximations are constructed [2]. We can easily illustrate this situation on the simple example of 1-D ordinary differential equations of second order. We consider the real functions $s(x)=x /(\exp (x)-1)>0, s^{\prime}(x)=\frac{d s}{d x}<0\left(s(0)=1, s^{\prime}(0)=-0.5\right)$ with the Patankar approximations [6] in the following form:

$$
\begin{aligned}
s(x) & =\max \left((1-0.1|x|)^{5}, 0\right)+\max (-x, 0), \\
s^{\prime}(x) & =-0.5+0.5 \operatorname{signum}(x)\left(1-\max (1-0.1|x|, 0)^{4}\right) .
\end{aligned}
$$


1. For the 1-D part $(x=r)$ of the first equation (4.8) we obtain the ordinary differential equation

$$
\left(b u^{\prime}\right)^{\prime}+a u^{\prime}=\tilde{f}
$$

where $u=\omega, b=r^{3}, a=-\operatorname{Re} r^{3} v_{r}, \tilde{f}=r^{3} f, f$ is the other part of the first equations of (4.8).

The monotonic approximations of (7.2) have the form $[2,4]$

$$
b_{i+0.5} s\left(-\alpha_{i+0.5} h_{1}\right)\left(u_{i+1}-u_{i}\right)-b_{i-0.5} s\left(\alpha_{i-0.5} h_{1}\right)\left(u_{i}-u_{i-1}\right)=h_{1}^{2} \tilde{f}_{i}
$$

where $i=\overline{1, n_{r}}, \alpha=a / b=-\operatorname{Re} v_{r}, g_{i \pm 0.5}=g\left(x_{i \pm 0.5}\right), x_{i \pm 0.5}=x_{i} \pm h_{1} / 2, g=\alpha ; b$.

For the 1-D part $(x=r)$ of the first equation of (4.8) we obtain the equations (7.2), (7.3) with $u=W, b=r^{-1}, a=-\operatorname{Re} r^{-1} v_{r}, \tilde{f}=r^{-1} f$.

For the 1-D part $(x=r)$ of equation (5.4) we obtain the equations (7.2), (7.3) with $u=T, b=r, a=-P e r v_{r}, \tilde{f}=r f$.

For the 1-D part $(x=z)$ of equation (5.4) we obtain the equations (7.2) with $u=T, b=$ $1, a=-P$ e $v_{z}, \tilde{f}=f$ and the finite-difference equations

$$
b_{j+0.5} s\left(-\alpha_{j+0.5} h_{2}\right)\left(u_{j+1}-u_{j}\right)-b_{j-0.5} s\left(\alpha_{j-0.5} h_{2}\right)\left(u_{j}-u_{j-1}\right)=h_{2}^{2} \tilde{f}_{j},
$$

where $j=\overline{2, n_{z}}, \alpha=-\operatorname{Pe} v_{z}, g_{j \pm 0.5}=g\left(x_{j \pm 0.5}\right), x_{j \pm 0.5}=x_{j} \pm h_{2} / 2, g=\alpha ; b$.

2. For the 1-D part $(x=z)$ of the first and second equations of (4.8) we obtain system of two ordinary differential equations

$$
\left\{\begin{aligned}
c \omega^{\prime \prime}+d \omega^{\prime}+c W^{\prime} & =f_{1}, \\
W^{\prime \prime}+d W^{\prime} & =f_{2},
\end{aligned}\right.
$$

where $d=-\operatorname{Rev}_{z}, c=2 \operatorname{Re} \Gamma^{2} W r^{-4}, f_{1}, f_{2}$ are the other parts of equations (4.8).

The corresponding monotonic finite-difference equations are obtained in the following form [2]:

$$
\begin{aligned}
s\left(-d_{j+0.5} h_{2}\right)\left(\omega_{j+1}-\omega_{j}\right) & -s\left(d_{j-0.5} h_{2}\right)\left(\omega_{j}-\omega_{j-1}\right) \\
& -h_{2}\left(c_{j+0.5} s^{\prime}\left(-d_{j+0.5} h_{2}\right)\left(W_{j+1}-W_{j}\right)\right. \\
& \left.+c_{j-0.5} s^{\prime}\left(d_{j-0.5} h_{2}\right)\left(W_{j}-W_{j-1}\right)\right)=h_{2}^{2} f_{1, j}, \\
s\left(-d_{j+0.5} h_{2}\right)\left(W_{j+1}-W_{j}\right) & -s\left(d_{j-0.5} h_{2}\right)\left(W_{j}-W_{j-1}\right)=h_{2}^{2} \tilde{f}_{2, j},
\end{aligned}
$$

where $j=\overline{2, n_{z}}, W_{j \pm 0.5}=0.5\left(W_{j}+W_{j \pm 1}\right)$. 


\section{The finite-difference scheme for MHD equations}

For the first and second equations of (4.8) we obtain from (7.3), (7.6) the following finitedifference equations of the second order of approximation:

$$
\begin{aligned}
\frac{1}{h_{1}^{2} r_{i}^{3}}\left(r _ { i + 0 . 5 } ^ { 3 } s r _ { i , j } ^ { + } \left(\omega_{i+1, j}\right.\right. & \left.-\omega_{i, j}\right)-r_{i-0.5}^{3} s r_{i, j}^{-}\left(\omega_{i, j}-\omega_{i-1, j}\right) \\
& +\frac{1}{h_{2}^{2}}\left(s z_{i, j}^{+}\left(\omega_{i, j+1}-\omega_{i, j}\right)-s z_{i, j}^{-}\left(\omega_{i, j}-\omega_{i, j-1}\right)\right) \\
& -2 R e \Gamma^{2} \frac{1}{h_{2} r_{i}^{4}}\left(W_{i, j+0.5} s 1 z_{i, j}^{+}\left(W_{i, j+1}-W_{i, j}\right)\right. \\
& \left.+W_{i, j-0.5} s 1 z_{i, j}^{-}\left(W_{i, j}-W_{i, j-1}\right)\right)=-\frac{R e . T e}{r_{i}} f_{i, j}^{\phi}, \\
\frac{r_{i}}{h_{1}^{2}}\left(r _ { i + 0 . 5 } ^ { - 1 } s r _ { i , j } ^ { + } \left(W_{i+1, j}\right.\right. & \left.\left.-W_{i, j}\right)-r_{i-0.5}^{-1} s r_{i, j}^{-}\left(W_{i, j}-W_{i-1, j}\right)\right) \\
& +\frac{1}{h_{2}^{2}}\left(s z_{i, j}^{+}\left(W_{i, j+1}-W_{i, j}\right)-s z_{i, j}^{-}\left(W_{i, j}-W_{i, j-1}\right)\right)=0,
\end{aligned}
$$

where

$$
\begin{aligned}
s z_{i, j}^{ \pm} & =s\left( \pm \operatorname{Re}\left(v_{z}\right)_{i, j \pm 0.5} h_{2}\right), \\
s 1 z_{i, j}^{ \pm} & =s^{\prime}\left( \pm \operatorname{Re}\left(v_{z}\right)_{i, j \pm 0.5} h_{2}\right), \\
s r_{i, j}^{ \pm} & =s\left( \pm \operatorname{Re}\left(v_{r}\right)_{i \pm 0.5, j} h_{1}\right), \\
\left(v_{z}\right)_{i, j \pm 0.5} & =\left(\psi_{i+1, j \pm 1}-\psi_{i-1, j \pm 1}+\psi_{i+1, j}-\psi_{i-1, j}\right) /\left(4 r_{i} h_{1}\right), \\
\left(v_{r}\right)_{i \pm 0.5, j} & =-\left(\psi_{i \pm 1, j+1}-\psi_{i \pm 1, j-1}+\psi_{i, j+1}-\psi_{i, j-1}\right) /\left(4 r_{i \pm 0.5} h_{2}\right) .
\end{aligned}
$$

For the third equation of (4.8) we obtain the finite-difference equation with central differences

$$
\delta_{z}^{2} \psi_{i, j}+\delta_{r}^{2} \psi_{i, j}-r_{i}^{-1} \delta_{r} \psi_{i, j}=-r_{i}^{2} \omega_{i, j}
$$

where $\delta_{z}^{2} \psi_{i, j}=h_{2}^{-2}\left(\psi_{i, j+1}-2 \psi_{i, j}+\psi_{i, j-1}\right), \quad \delta_{r}^{2} \psi_{i, j}=h_{1}^{-2}\left(\psi_{i+1, j}-2 \psi_{i, j}+\psi_{i-1, j}\right)$ are the spacecentered differences of second order for the approximation of the derivatives $\frac{\partial^{2} \psi}{\partial z^{2}}, \frac{\partial^{2} \psi}{\partial r^{2}}, \delta_{r} \psi_{i, j}=$ $0.5 h_{1}^{-1}\left(\psi_{i+1, j}-\psi_{i-1, j}\right)$ is the space-centered difference of first order for the approximation of the derivative $\frac{\partial \psi}{\partial r}$.

For the heat transport equation (5.4), from (7.3) we obtain the finite-difference equations

$$
\begin{aligned}
\frac{1}{h_{1}^{2} r_{i}}\left(r_{i+0.5} \tilde{s} r_{i, j}^{+}\left(T_{i+1, j}-T_{i, j}\right)\right. & \left.-r_{i-0.5} \tilde{s} r_{i, j}^{-}\left(T_{i, j}-T_{i-1, j}\right)\right)+\frac{1}{h_{2}^{2}}\left(\tilde{s} z_{i, j}^{+}\left(T_{i, j+1}-T_{i, j}\right)\right. \\
& \left.-\tilde{s} z_{i, j}^{-}\left(T_{i, j}-T_{i, j-1}\right)\right)+\operatorname{Ec} \operatorname{Pr} \Phi_{i, j}+K_{T} q_{i, j}=0
\end{aligned}
$$

where

$$
\begin{aligned}
\tilde{s} z_{i, j}^{ \pm}= & s\left( \pm P e\left(v_{z}\right)_{i, j \pm 0.5} h_{2}\right), \\
\tilde{s} r_{i, j}^{ \pm}= & s\left( \pm P e\left(v_{r}\right)_{i \pm 0.5, j} h_{1}\right), \\
\Phi_{i, j}= & 2\left(\left(r_{i}^{-2} \delta_{z} \psi_{i, j}-r_{i}^{-1} \delta_{z r}^{2} \psi_{i, j}\right)^{2}+\left(r_{i}^{-1} \delta_{z r}^{2} \psi_{i, j}\right)^{2}+\left(r_{i}^{-2} \delta_{z} \psi_{i, j}\right)^{2}\right)+\left(r_{i} \omega_{i, j}+2 r_{i}^{-1} \delta_{z}^{2} \psi_{i, j}\right)^{2} \\
& +\Gamma^{2}\left(\left(r_{i}^{-1} \delta_{z} W_{i, j}\right)^{2}+\left(r_{i}^{-1} \delta_{r} W_{i, j}-2 r_{i}^{-2} W_{i, j}\right)^{2}\right)
\end{aligned}
$$

is the discrete form for the dissipation function $\Phi, \delta_{z} g_{i, j}=0.5 h_{2}^{-1}\left(g_{i, j+1}-g_{i, j-1}\right)$ is the spacecentered difference of first order for the approximation of the derivative $\frac{\partial g}{\partial z}, g=\psi ; W, \delta_{z r}^{2} \psi_{i, j}=$ 
$0.25\left(h_{1} h_{2}\right)^{-1}\left(\psi_{i+1, j+1}+\psi_{i-1, j-1}-\psi_{i+1, j-1}-\psi_{i-1, j+1}\right)$ is the space-centered difference of second order for the approximation of the derivative $\frac{\partial^{2} \psi}{\partial r \partial z}$.

For the boundary conditions we have the following approximations:

1) if $z=0$, then $\psi_{i, 1} ; W_{i, 1}$ are given in the inlet and $\omega_{i, 1}=T_{i, j}=0, i=\overline{1, n_{r}}$,

2) if $z=l$, then $g_{i, n_{z}+1}=g_{i, n_{z}}$ for $g=\psi ; \omega ; W ; T, i=\overline{1, n_{r}}$,

3) if $r=1$, then $\omega_{n n, j}$ is given by (6.4) and $\psi_{n n, j}=2 \psi_{w}-\psi_{n_{r}, j}, W_{n n, j}=W_{n_{r}, j}, T_{n n, j}=$ $T_{n_{r}, j}-0.5 h_{1} \operatorname{Bi}\left(T_{n n, j}+T_{n_{r}, j}\right)$, where $n n=n_{r}+1, \psi_{w}=0.5\left(r_{1}^{2}+\beta\left(1-r_{1}^{2}\right)\right), j=\overline{2, n_{z}}$,

4) if $r=0$, then $g_{0, j}=g_{1, j}$ for $g=\omega ; T$, and $g_{0, j}=-g_{1, j}$ for $g=\psi ; W, j=\overline{2, n_{z}}$.

\section{The numerical method}

The finite difference scheme (8.1)-(8.4) is calculated by the relaxation method in the following form:

$$
g_{i, j}^{(n)}=\Omega_{g} g_{i, j}^{z}+\left(1-\Omega_{g}\right) g_{i, j}^{(n-1)},
$$

where $n=1,2,3, \ldots$ is the iteration number, $\Omega_{g}$ represents the relaxation parameters, $g_{i, j}^{z}$ is the $\mathrm{n}$-th approximation by Seidel's iteration technique, $i=\overline{1, n_{r}}, j=\overline{2, n_{z}}, g=\psi ; \omega ; W ; T$. The solutions converged when they satisfied the following convergence criterion:

$$
\max \left|\frac{g^{(n)}-g^{(n-1)}}{g^{(n)}}\right|<10^{-2} .
$$

It is difficult to find the optimal values of the up-relaxation parameter $\Omega_{o}$ for $\Omega_{\psi}, \Omega_{T}$ in cylindrical coordinates, hence we will apply the quantity taken from the approximation of Poisson's equation in the Descartes coordinates:

$$
\Omega_{o}=\frac{2}{1+\sqrt{1-\eta_{0}^{2}}}
$$

where $\eta_{0}=0.5\left(\cos \left(\pi / n_{r}\right)+\cos \left(\pi / n_{z}\right)\right)$.

For instance, if $n_{r}=10, n_{z}=20, l=2$, then from (9.2) it follows, that $\Omega_{o}=1.6$, but numerical experiments show that the fastest convergence on such a grid is for $\Omega_{0}=1.5$ (approximately 200 iterations; if $\Omega_{o}=1$ (Seidel's iterations), then $n=2000$ ).

Others are the under-relaxation parameters in the form $\Omega_{\omega}=0.5, \Omega_{W}=0.5$.

\section{Numerical results}

As the basis for carrying out calculations $N$ circular conductors are selected, which are arranged in the axial direction at the points $z_{i}=0.2 i, i=\overline{1, N}$. The results of numerical experiments for $\left\langle F_{r}\right\rangle,\left\langle F_{z}\right\rangle,\left\langle f_{\phi}\right\rangle,\left\langle j_{\phi}\right\rangle$ in the dimensionless form $0.5 S_{N}^{r}, 0.5 S_{N}^{z}, 0.5 S_{N}^{\phi}, 0.5 S_{N}^{Q}$ and $\psi, \omega, W, T$ was performed at the University of Latvia; they were obtained with the aid of the computer program MAPLE in the case of electric current with different phases $\theta=\pi / 3 ; 2 \pi / 3 ; \pi$, and $l=2, h_{1}=h_{2}=0.1, r_{1}=0.5, \beta=$ $0.1, j_{0}=10^{6} \frac{A}{m^{2}}, N=6$.

The numerical results show that the force fields induced by alternating current as well as the heat source are concentrated on the surface of the cylinder in the vicinity of the circular 
electrodes and that the conducting material is heated best on the cylindrical surface after the last electrode. The results depend on the arrangement of electrodes and on the phase shift. If the parameter $\Gamma$ is increased, inside the cylinder one vortex appears, which, starting from $\Gamma>4$, develops into a vortex system, which exerts a strong influence on the temperature distribution (the electrolyte in the cylinder is heated more rapidly).

Figures 1-14 show typical results of the calculations: the magnetic field and the distribution of heat sources rises to a maximum on the surface of the cylinder. We can see the vortex formation and its temperature field depending on the parameters $R e, T e$, and $\Gamma$.

Without axial streams $\left(v_{r}=v_{z}=v_{\phi}=0\right)$ we obtain the following results for the dimensionless values of $\left\langle f_{\phi}\right\rangle, q,\left\langle F_{r}\right\rangle,\left\langle F_{z}\right\rangle$, T for different phases $\theta$ and distributions $z j=\left[z_{1}, z_{2}, z_{3}, z_{4}, z_{5}, z_{6}\right]$ of the electric current in the conductors the following results:

1) $\theta=\pi / 3, z j=[0.2,0.4,0.6,0.8,1.0,1.2]$ (the conductors are connected normally one after another $),<f_{\phi}>\in(-7.1,236.0), q \in(0,5.6),<F_{r}>\in(-28.4,28.4),<F_{z}>\in$ $(-39.4,3.8), T_{\max }=1.6$;

2) $\theta=\pi / 3, z j=[0.2,0.6,1.0,0.4,0.8,1.2]$ (the conductors are connected to each other skipping one of them), $<f_{\phi}>\in(-43.0,20.0), q \in(0,1.3),<F_{r}>\in(-12.5,12.5),<$ $F_{z}>\in(-11.5,10.8), T_{\max }=0.2$;

3) $\theta=2 \pi / 3, z j=[0.2,0.4,0.6,0.8,1.0,1.2]$ (the conductors are connected normally one after another $),<f_{\phi}>\in(-0.3,126.0), q \in(0,2.3),<F_{r}>\in(-8.4,11.3),<F_{z}>\in$ $(-16.7,1.0), T_{\max }=0.3$;

4) $\theta=\pi, z j=[0.2,0.6,0.4,0.8,1.0,1.2]$ (the conductors are connected skipping one of them) $,<f_{\phi}>=0, q \in(0,5.0),<F_{r}>=<F_{z}>=0, T_{\max }=0.5$;

5) $\theta=\pi, z j=[0.2,1.2,0.4,1.0,0.6,0.8]$ (the conductors are connected symmetrically in pairs the first the sixth, the second with the fifth, the third with the fourth), $\left\langle f_{\phi}\right\rangle=$ $0, q \in(0,8.8),<F_{r}>=<F_{z}>=0, T_{\max }=2.0$;

6) $\theta=\pi, z j=[0.2,0.4,0.6,0.8,1.0,1.2]$ (the conductors are connected normally one after another $),<f_{\phi}>=0, q \in(0,1.8),<F_{r}>=<F_{z}>=0, T_{\max }=0.2$.

As can be seen, in the $5^{\text {th }}$ variant (two-phase current) we obtain the highest temperature (four times higher than in the $4^{\text {th }}$ variant and ten times that of the $6^{\text {th }}$ variant), although the averaged forces cancel.

In the case of a hydrodynamic flow $\left(R e=500, \Gamma=2, T e=0.1, K_{T}=0.5\right)$, we have the following results:

1) $\psi_{\max }=0.156, \omega \in(-14.7,3.95), W_{\max }=0.57, T_{\max }=0.067$ for $\theta=\pi, z j=$ $[0.2,1.2,0.4,1.0,0.6,0.8]$

2) $\psi_{\max }=0.256, \omega \in(-12.6,21.9), W_{\max }=0.73, T_{\max }=0.087$ for $\theta=\pi / 3, z j=$ $[0.2,0.4,0.6,0.8,1.0,1.2$.

\section{Conclusions}

1. Squirt motion of a weakly electrically conducting fluid influenced by an alternating electromagnetic field in a finite cylinder is investigated. 
2. A real variable approach is used to describe time-averaged electromagnetic forces and fields.

3. An original monotonic difference scheme for approximation of this mathematical model is developed.

4. Numerical results show that the maximum temperature distribution is strongly influenced by the connection method used by producers of electrical energy as also by the distribution of vortices in the cylinder.

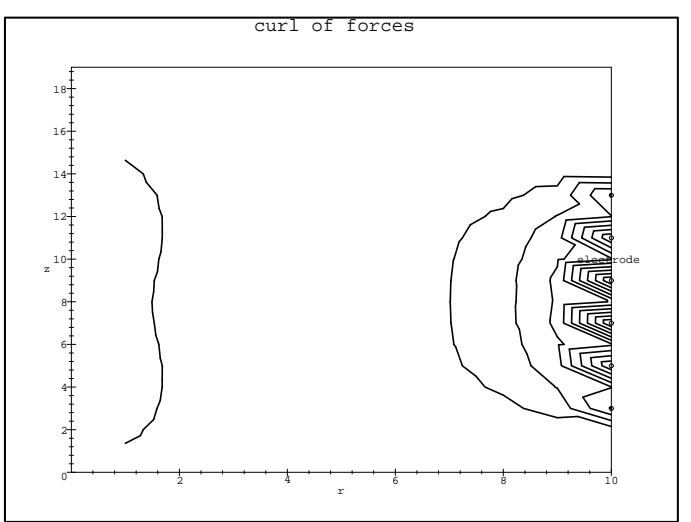

Figure 1. Distribution of the azimuthal component $<\operatorname{rot}_{\phi}>F \in(-7.1,236.0)$

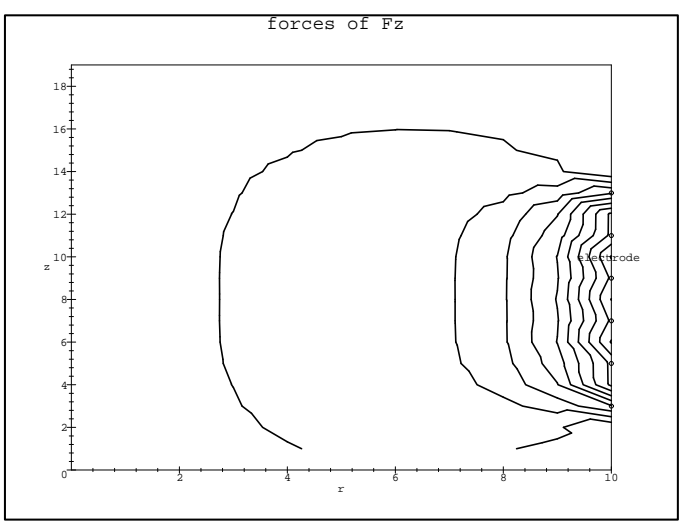

Figure 3. Distribution of the axial component $<F_{z}>\in(-39.4,3.7)$

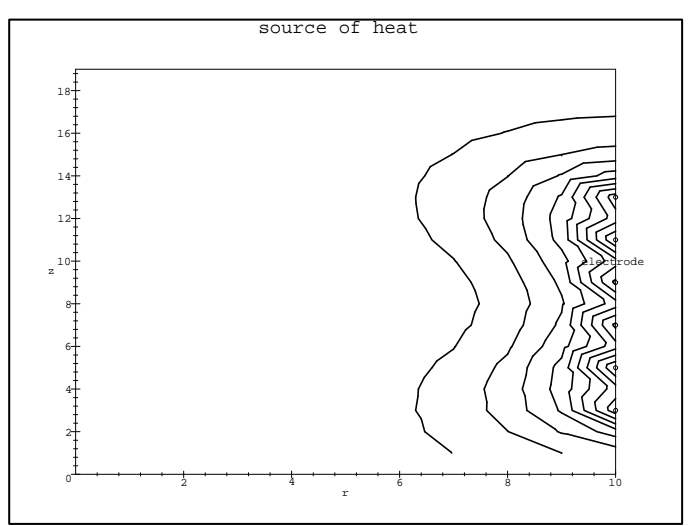

Figure 2. Distribution of the source term $q \in$ $(0,0.5)$

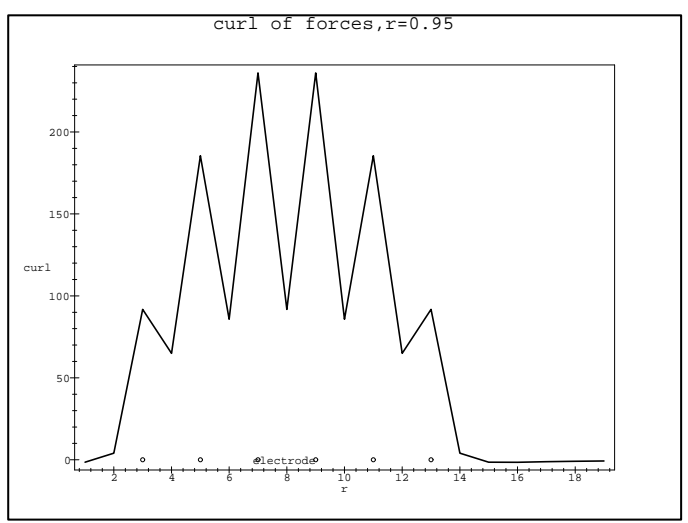

Figure 4. Distribution of the azimuthal component $\left\langle\operatorname{rot}_{\phi} F>\right.$ for $r=0.95$ 


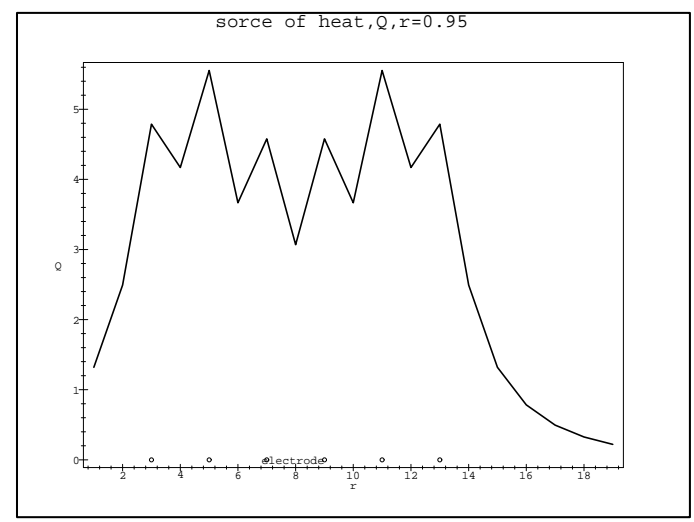

Figure 5. Distribution of the source term $q$ for $r=0.95$

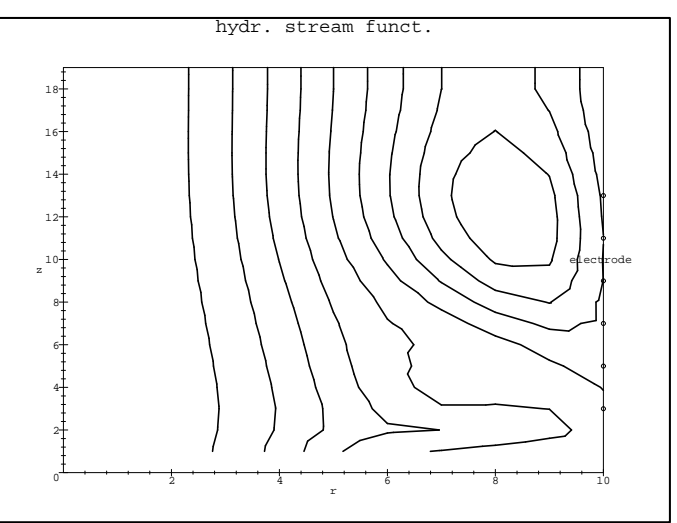

Figure 7. Distribution of the stream function $\psi \in(0,0.265), R e=500, \quad \Gamma=2, T e=0.1$

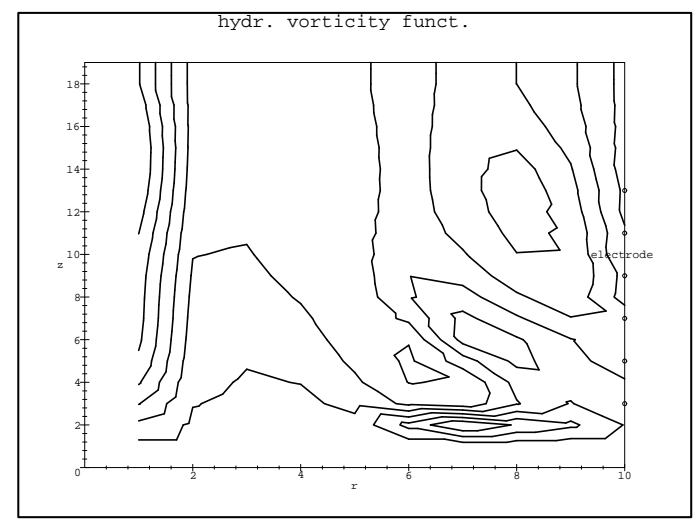

Figure 6. Distribution of the vorticity function $\omega \in(-12,22), R e=500, \Gamma=2, T e=0.1$

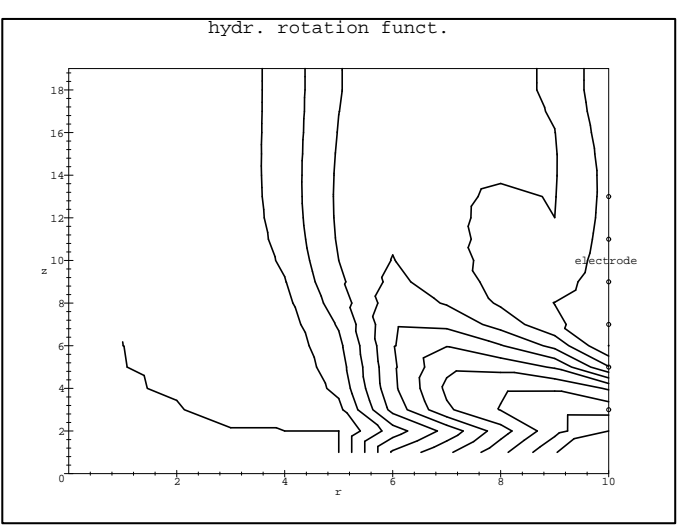

Figure 8. Distribution of the circulation $W \in$ $(0,0.73), R e=500, \Gamma=2, T e=0.1$ 


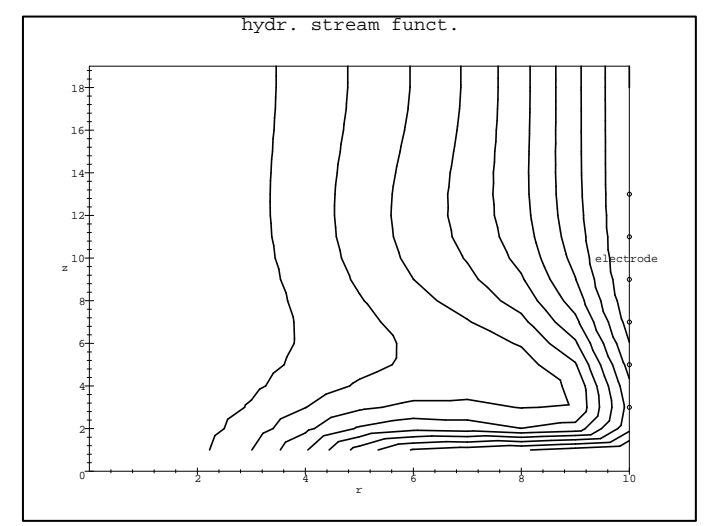

Figure 9. Distribution of the stream function $\psi \in(0,0.16), R e=500, \Gamma=2, \quad T e=0$

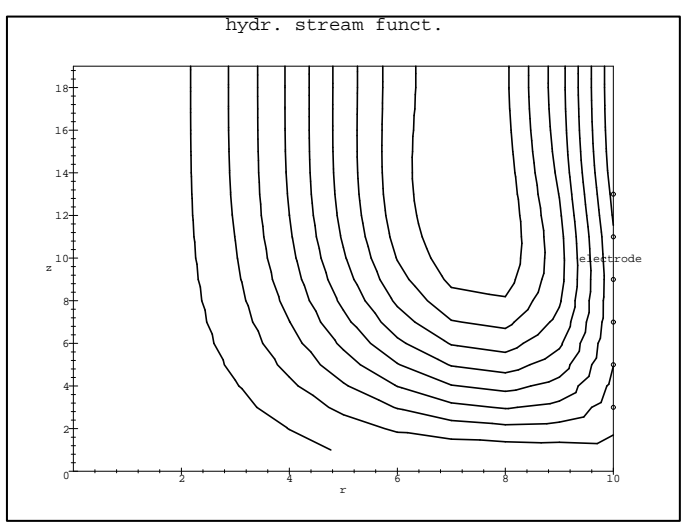

Figure 11. Distribution of the stream function $\psi \in(0,0.92), R e=500, \quad \Gamma=2, T e=1$

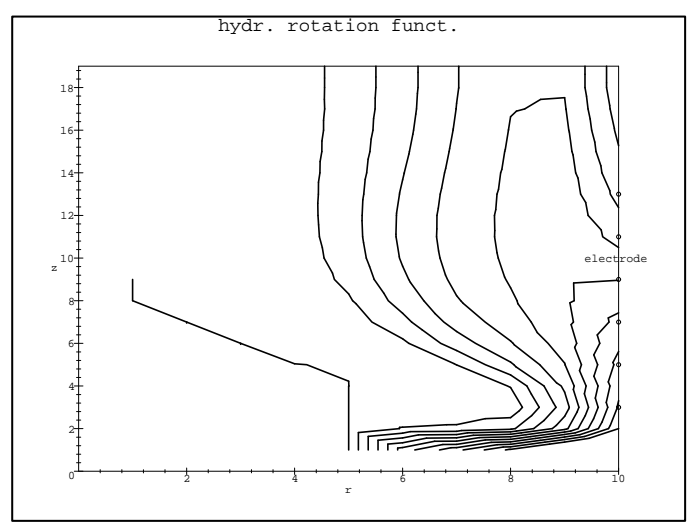

Figure 10. Distribution of the rotation $W \in$ $(0,0.55), R e=500, \Gamma=2, T e=0$

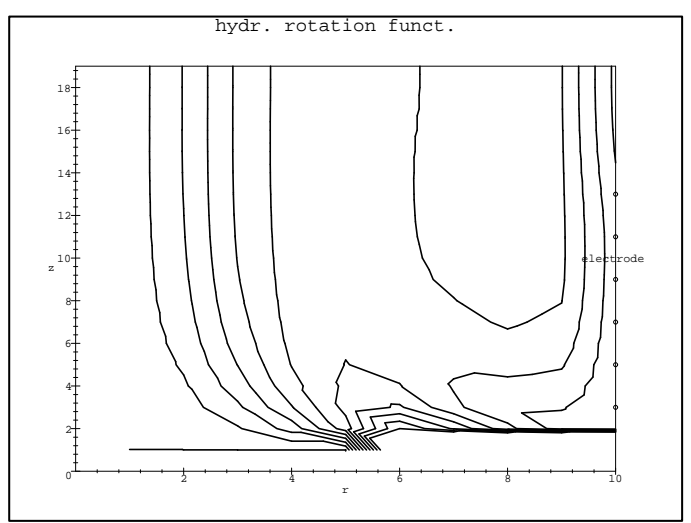

Figure 12. Distribution of the rotation $W \in$ $(0,0.19), R e=500, \Gamma=2, T e=1$ 


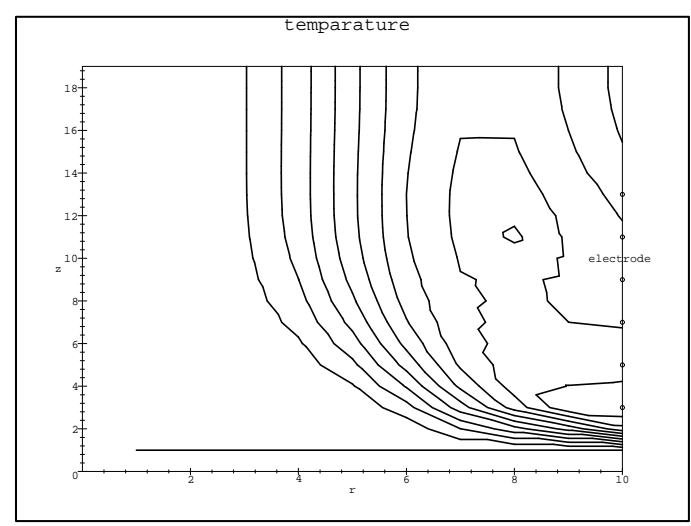

Figure 13. Distribution of the temperature $T \in$ $(0,0.22), R e=500, \Gamma=2, \quad T e=0.1$

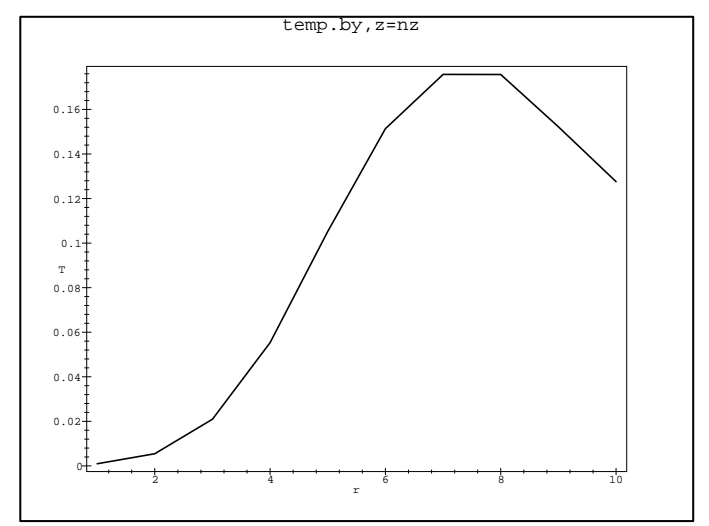

Figure 14. Distribution of the temperature $T$ for $z=1.9$

\section{References}

[1] B. L. Alijevskij and V. 1. Orlov, Calculation of the Parameters of Magnetic Fields for Axially-Symmetric Inductors, Moskow, 1983, in Russian.

[2] H. Kalis, Special computational methods for solution of MHD problems, MHD, 30 (1994), No. 2, pp. $144-155$, in Russian.

[3] H. Kalis, J. Kolesnikov, and N. Polakov, Investigation of a rotating flow in a transverse magnetic field, MHD, (1983), No. 1, pp. 71-76, in Russian.

[4] J. Krumins and Y. Gelfgat, Calculation of the distribution of electromagnetic fields and forces induced by a rotating magnetic field in a conducting cylinder of finite length, MHD, 34 (1998), No. 3, pp. 241-262, in Russian.

[5] J. P. Narain, Numerical prediction of confined swirling jets, Computers and Fluids, 5 (1977), pp. 115-125.

[6] S. Patankar, Calculation of the Heat Transfer and Fluid Flow Problems, Moskow, 1984, in Russian. 